\section{Uso de álcool, tabaco e outras drogas por adolescentes escolares em município do Sul do Brasil}

\author{
Alcohol, tobacco, and other drug use by teenage \\ students in a city in Southern Brazil
}

\footnotetext{
1 Programa de Pós-graduação em Saúde Coletiva, Universidade Luterana do Brasil, Canoas, Brasil. 2 Secretaria Municipal de Educação de Gravataí, Gravataí, Brasil.

Correspondência P. C. Vieira

Programa de Pós-graduação em Saúde Coletiva Universidade Luterana do Brasil.

Rua Luiz Afonso 307, apto. 1102, Porto Alegre, RS 90050-310, Brasil.

patriciaconzatti@terra.com.br
}

\begin{abstract}
This study analyzes alcohol, tobacco, and other drug use by seventh-graders in municipal schools in the city of Gravataí, Rio Grande do Sul State, Brazil, and associated factors. A cross-sectional school survey was conducted in a representative random sample of 1,170 seventh-graders from municipal schools. The bivariate Cox regression model, modified for cross-sectional studies, was used to evaluate associations between specific factors and outcomes. Prevalence rates for lifetime consumption of alcohol, tobacco, and other drugs were $60.7 \%, 16.9 \%$, and $2.4 \%$, respectively. Consumption rates in the previous 30 days were: alcohol, 33\%; tobacco, 4.4\%; and other drugs, $0.6 \%$. The study showed an association with substance use by family and friends. Prevalence of tobacco and alcohol use during the previous 30 days was associated with feelings of sadness, loneliness, insomnia, and suicidal ideation. Use of other drugs was associated with loneliness and suicidal ideation.
\end{abstract}

Adolescent Behavior; Alcoholic Beverages; Tobacco; Street Drugs

\author{
Patrícia Conzatti Vieira ${ }^{1}$ \\ Denise Rangel Ganzo de Castro Aerts 1 \\ Sílvia Letícia Freddo 1 \\ Alex Bittencourt 1 \\ Lisiane Monteiro 2
}

\section{Introdução}

A adolescência é uma fase da vida do ser humano caracterizada por mudanças biológicas, cognitivas, emocionais e sociais, constituindo-se em importante momento para a adoção de novas práticas, comportamentos e ganho de autonomia ${ }^{1}$. É um período marcado pela indecisão ${ }^{2}$, sinalizando a passagem da infância protegida para a exposição à vida adulta. A tendência grupal e a evolução da sexualidade também são aspectos importantes, havendo, às vezes, um descompasso entre a acelerada mudança corporal e a maturidade psíquica 1 , o que pode contribuir para a insatisfação do adolescente com seu próprio corpo.

Nessa fase, estimulado pelas intensas transformações, o adolescente torna-se mais vulnerável a comportamentos que podem fragilizar sua saúde, como alimentação inadequada, sedentarismo, tabagismo, consumo de álcool e de drogas ${ }^{3}$. O uso dessas substâncias pelos pais e amigos, assim como o desenvolvimento de sintomas depressivos, são fatores de risco para a experimentação e abuso de drogas pelo adolescente ${ }^{4}$. Na busca de sua própria identidade, o jovem, muitas vezes, adota comportamentos dos adultos, cabendo aos familiares apresentarem-se como modelos saudáveis 5 . No entanto, além da vulnerabilidade individual, devido às características próprias de seu desenvolvimento 1, os adolescentes também estão expostos à 
vulnerabilidade institucional 6 , em virtude da escassez de ações voltadas diretamente para essa faixa etária e da carência de profissionais de saúde especializados para o atendimento de suas necessidades. Da mesma forma, evidenciase a vulnerabilidade social, quando a sociedade cobra do adolescente o desenvolvimento da maturidade e a entrada no sistema produtivo 7 .

Anualmente, 1,7 milhão de adolescentes no mundo perde a vida, a maioria por acidente de trânsito, suicídio e homicídio ${ }^{8}$, muitas vezes associados ao consumo de álcool ou de outras drogas. No inquérito nacional realizado pelo Centro Brasileiro de Informações sobre Drogas Psicotrópicas (CEBRID), em 2004, os escolares dos ensinos fundamental e médio de 27 capitais brasileiras apresentaram 23,5\% de prevalência de uso de drogas, exceto álcool e tabaco, no sexo masculino, e $21,7 \%$, no sexo feminino ${ }^{9}$. No ano de 1995, Pechanski \& Barros 10 identificaram que, entre jovens de 10 a 18 anos, moradores de Porto Alegre, Rio Grande do Sul, Brasil, 71\% haviam experimentado bebida alcoólica, chegando a quase $100 \%$ na idade de 18 anos.

Em inquérito realizado sobre tabagismo entre escolares de 7ạ e 8a séries do Ensino Fundamental (atualmente 8o e 9o anos) e da la série do Ensino Médio (atualmente 1o ano), em 12 capitais brasileiras, foi encontrado alto percentual de experimentação de cigarros entre 13 e 15 anos, variando de $36 \%$ no sexo masculino e $31 \%$ no feminino, em Vitória, Espírito Santo, Brasil, e de $58 \%$ no masculino e $50 \%$ no feminino, em Fortaleza, Ceará, Brasil. Em Porto Alegre, 20\% fumavam cigarros no momento da pesquisa e $7 \%$ consumiam outro tipo de produto derivado do tabaco, além dos cigarros 11. Os problemas começam com o uso esporádico de álcool, tabaco e outras drogas, passando a ser freqüente ainda nesse período de vida ou na fase adulta 12 .

Assim, como uma forma de conhecer a população em estudo e sugerir ações de saúde dirigidas para a prevenção do uso dessas substâncias, esta pesquisa teve como objetivo estudar o comportamento dos estudantes da 7 a série do ensino público municipal de Gravataí, Rio Grande do Sul, Brasil, em relação ao consumo de álcool, tabaco e outras drogas, como também avaliar a associação com características familiares, do grupo de amigos, estado nutricional e aspectos emocionais dos jovens, no ano de 2005.

\section{Material e método}

Foi realizado um estudo transversal, na cidade de Gravataí, que, em 2005, apresentava uma população estimada de 232.446 habitantes (Instituto
Brasileiro de Geografia e Estatística; http:/ /www. ibge.gov.br, acessado em Jun/2005). A rede de ensino público municipal é formada por 66 escolas urbanas e rurais, das quais 61 são de Ensino Fundamental diurno, sendo a segunda maior rede do estado (Prefeitura Municipal de Gravataí. http:// www.gravatai.rs.gov.br, acessado em Jun/2005).

A população-alvo foi composta por alunos matriculados na 7a série das escolas públicas municipais de Ensino Fundamental. Optou-se por escolher este grau de estudo em virtude de a maioria dos escolares encontrarem-se na faixa etária preconizada pela Organização Mundial da Saúde (OMS - 13 a 15 anos) 13 para monitorização de fatores de risco em adolescentes, o que é acatado pelo Ministério da Saúde 3 .

Em março de 2005, havia 2.282 alunos matriculados na 7a série. Tomou-se como ponto de partida para o cálculo da amostra essa população, distribuída de forma não homogênea em 15 regiões do município. Utilizando-se uma prevalência de $50 \%$ para os desfechos de interesse, um erro máximo de $+3 \%$ e um nível de significância de 0,05, estimou-se uma amostra composta por 728 estudantes. Aplicando-se um efeito delineamento de 1,5, o tamanho da amostra passou para 1.092 alunos, que foi acrescido de $20 \%$ para suprir possíveis perdas. Considerando que 1.312 escolares representavam cerca de metade da população-alvo, optou-se por sortear um número de turmas equivalente à metade mais uma das turmas de 7a série existentes em cada região. Com isso, ao final da seleção, obteve-se uma amostra com 1.366 alunos. Destes, 31 recusaram-se a responder ao questionário, 30 não foram autorizados pelos pais, 105 trocaram de escola, 14 não compareceram nos dias da aplicação do questionário, 1 foi expulso da escola, 3 eram alunos infreqüentes e 12 não responderam por evasão. Assim, a amostra final foi de 1.170 estudantes, sendo 34 alunos de escolas da área rural.

Os dados foram coletados em sala de aula por meio de questionário, com perguntas fechadas baseadas no instrumento elaborado pela OMS 14 para investigação da saúde do escolar. Esse questionário, além de perguntas relacionadas ao uso do álcool, tabaco e outras drogas (maconha, cocaína, crack, ecstasy, solventes, anfetaminas e anabolizantes), continha questões para identificar fatores em estudo, como sentimento de tristeza e solidão; dificuldade de dormir e ideação suicida nos últimos 12 meses; uso das substâncias pelos pais e amigos. As variáveis sexo (masculino e feminino), idade, cor da pele auto-referida (branca e não branca) e classificação sócio-econômica foram coletadas em outro questionário, especialmente constru- 
ído para esse fim, sendo utilizado o critério da Associação Brasileira de Empresas de Pesquisa (ABEP. Critério de Classificação Econômica Brasil. http://www.abep.org/codigosguias/ABEP_ CCEB.pdf, acessado em Jan/2006) para a classificação sócio-econômica.

Para obtenção dos dados antropométricos, os escolares foram avaliados em relação ao peso e à altura. Para a pesagem, foram utilizadas balanças digitais (Seca, Hamburgo, Alemanha) doadas pelo Fundo das Nações Unidas para a Infância (UNICEF), com capacidade para $150 \mathrm{~kg}$ e precisão de 50g. A altura foi aferida com auxílio de estadiômetro de metal (Wiso, São José, Brasil), com precisão em milímetros. As técnicas utilizadas são as recomendadas pela OMS 15. O estado nutricional foi avaliado pelo cálculo do índice de massa corporal $\left(\mathrm{IMC}=\right.$ peso/altura $\left.{ }^{2}\right)$, sendo utilizada a população de referência da distribuição percentilar proposta por Must et al. 16. Para os alunos com percentil > 50, foi utilizada, na seqüência, a classificação de Cole et al. 17, considerada como mais acurada para a detecção do sobrepeso e obesidade. Os escolares foram classificados em: baixo peso/desnutridos (IMC < percentil 15); eutróficos (IMC > percentil 15 e $<$ percentil 85) e sobrepeso/obesidade (IMC > 85) 18.

A percepção da imagem corporal foi avaliada com o auxílio do Body Shape Questionnaire (BSQ) 19, que é um questionário auto-aplicável, validado no Brasil por Cordás \& Castilho 20. É utilizado para avaliar a preocupação com o ganho de peso, a baixa estima relacionada à aparência física, o desejo da perda de peso e a insatisfação com o corpo. Segundo os autores 19 , o BSQ recebe uma pontuação que é classificada em quatro grupos: (1) não preocupados com a imagem corporal (<81 pontos), (2) levemente preocupados (81-110 pontos), (3) moderadamente preocupados (111140 pontos) e (4) extremamente preocupados (> 140 pontos). Para fins deste estudo, as categorias foram reagrupadas, sendo os escolares classificados em preocupados (grupos 3 e 4) e não preocupados (grupos 1 e 2) com sua imagem corporal.

Os fatores em estudo foram investigados de acordo com cada um dos três desfechos (álcool, tabaco e outras drogas), cuja freqüência de uso foi caracterizada em "pelo menos uma vez na vida" (uso na vida) e "no mês que antecedeu a pesquisa" (uso nos últimos trinta dias).

As associações de interesse foram testadas no programa Stata 6.0 (Stata Corp., College Station, Estados Unidos) com regressão de Cox bivariada modificada para estudos transversais 21 , sendo os resultados apresentados na forma de razão de prevalência (RP), com seus respectivos intervalos de 95\% de confiança (IC95\%) e níveis de significância.
Este trabalho faz parte de um estudo maior intitulado A Saúde do Escolar da Rede Pública Municipal de Gravataí, RS, que foi aprovado pelo Comitê de Ética em Pesquisa da Universidade Luterana do Brasil (protocolo 2004-375 H).

\section{Resultados}

A amostra constituiu-se por $52,5 \%$ de adolescentes do sexo feminino, 52,6\% com a cor da pele auto-referida como branca e idade variando de 12 a 18 anos, com a média em 14 anos (desviopadrão de $\pm 1,13$ anos). Entre o total de escolares estudados, $60,7 \%$ relataram que haviam feito uso de bebida alcoólica pelo menos uma vez na vida. O tabaco foi experimentado por $16,9 \%$ dos adolescentes e outras drogas por 2,4\%. Quanto ao uso nos últimos trinta dias, o álcool também foi a substância de maior prevalência (33\%), seguido pelo tabaco $(4,4 \%)$ e por outras drogas $(0,6 \%)$.

A idade média do primeiro contato com tabaco e álcool, relatado pelos jovens, foi de 11,7 anos (desvio-padrão de $\pm 1,94$ anos) e 11,3 anos (desvio-padrão de $\pm 2,16$ anos), respectivamente. A experiência com outras drogas aconteceu mais tarde, com idade média de 13,0 anos (desvio-padrão de $\pm 1,43$ anos) (Tabela 1). Entre os 198 adolescentes que fizeram uso de tabaco pelo menos uma vez na vida, $109(55,1 \%)$ relataram não ter mais fumado e 146 (73,7\%) mencionaram não ter fumado nos últimos trinta dias. Entre os 52 que continuaram fumando, 31 (34,8\%) relataram fumar em casa e $24(46,1 \%)$ fumaram de 1 a 5 cigarros/dia no último mês. O uso de tabaco por pelo menos um dos pais foi referido por 58,6\% (116) dos alunos que haviam experimentado a substância, e 43\% (85) mencionaram que a maioria ou todos os amigos fumam (Tabela 2). Também foi evidenciado que o uso de tabaco pelos pais e pelos amigos associa-se com a experimentação dessa substância pelos adolescentes $(\mathrm{p}<0,003)$. A prevalência do uso de tabaco foi 1,5 (IC95\%: 1,14-1,91) e 4,7 (IC95\%: 3,09-7,03) vezes maior entre os jovens com pais e amigos fumantes, respectivamente, do que entre seus pares de referência. $\mathrm{O}$ uso de tabaco pelos amigos também se associou com o uso de outras drogas pelos jovens $(\mathrm{p}<0,002)$, mostrando que a freqüência do consumo dessas substâncias foi 5,4 (IC95\%: $1,63-17,65)$ vezes maior entre os escolares com amigos tabagistas.

Em relação aos 710 escolares que usaram álcool pelo menos uma vez na vida, 51\% (362) referiram ter experimentado em casa; $26,1 \%$ (185) ter bebido exageradamente pelo menos uma vez na vida e 47,9\% (340) não sabiam como seria a reação da família se chegassem alcoolizados em 
Distribuição dos escolares segundo o uso de tabaco, álcool e outras drogas em relação à idade da primeira experiência. Gravataí, Rio Grande do Sul, Brasil, 2005.

\begin{tabular}{|c|c|c|c|c|c|c|}
\hline \multirow[t]{2}{*}{ Variáveis } & \multicolumn{2}{|c|}{ Tabaco } & \multicolumn{2}{|c|}{ Álcool } & \multicolumn{2}{|c|}{ Outras drogas } \\
\hline & $\begin{array}{c}n \\
(\%)\end{array}$ & $\begin{array}{c}\text { Média } \\
\text { (desvio-padrão) }\end{array}$ & $\begin{array}{c}n \\
(\%)\end{array}$ & $\begin{array}{c}\text { Média } \\
\text { (desvio-padrão) }\end{array}$ & $\begin{array}{c}n \\
(\%)\end{array}$ & $\begin{array}{c}\text { Média } \\
\text { (desvio-padrão) }\end{array}$ \\
\hline Idade da primeira experiência (anos) & - & $11,7(1,94)$ & - & $11,3(2,16)$ & - & $13(1,43)$ \\
\hline Nunca experimentou & $972(8,31)$ & - & $460(39,3)$ & - & $1.142(97,6)$ & - \\
\hline$<10$ & $22(1,9)$ & - & $113(9,7)$ & - & - & - \\
\hline $10-12$ & $106(9,1)$ & - & $386(33,0)$ & - & $7(0,6)$ & - \\
\hline $13-15$ & $66(5,6)$ & - & $190(16,2)$ & - & $18(1,5)$ & - \\
\hline$\geq 16$ & $1(0,1)$ & - & $9(0,8)$ & - & $2(0,2)$ & - \\
\hline Ignorado & $3(0,3)$ & - & $12(1,0)$ & - & $1(0,1)$ & - \\
\hline Total & $1.170(100,0)$ & - & $1.170(100,0)$ & - & $1.170(100,0)$ & - \\
\hline
\end{tabular}

Tabela 2

Distribuição dos escolares que fizeram uso de tabaco na vida em relação ao local onde fuma, quantidade de cigarros/dia e uso de tabaco por pais e amigos. Gravataí, Rio Grande do Sul, Brasil, 2005.

\begin{tabular}{|c|c|c|}
\hline Variáveis & $\mathbf{n}$ & $\%$ \\
\hline \multicolumn{3}{|l|}{ Onde fuma } \\
\hline Não fuma & 109 & 55,1 \\
\hline Em casa & 31 & 15,7 \\
\hline Escola e outros & 12 & 6,0 \\
\hline Casa dos amigos & 23 & 11,6 \\
\hline Festas, bares, shopping, rua, restaurantes & 23 & 10,6 \\
\hline \multicolumn{3}{|l|}{ Quantidade de cigarros/dia nos últimos trinta dias } \\
\hline Não fumou & 146 & 73,7 \\
\hline Menos de 1 & 15 & 7,6 \\
\hline $1-5$ & 24 & 12,2 \\
\hline $6-20$ & 7 & 3,5 \\
\hline Mais de 20 & 6 & 3,0 \\
\hline \multicolumn{3}{|l|}{ Pais fumantes } \\
\hline Não & 80 & 40,4 \\
\hline Sim, o pai & 46 & 23,2 \\
\hline Sim, a mãe & 30 & 15,2 \\
\hline Sim, os dois & 40 & 20,2 \\
\hline Não sabe & 2 & 1,0 \\
\hline \multicolumn{3}{|l|}{ Amigos fumantes } \\
\hline Não & 24 & 12,1 \\
\hline Sim, a maioria & 80 & 40,5 \\
\hline Sim, poucos & 89 & 44,9 \\
\hline Sim, todos & 5 & 2,5 \\
\hline Total & 198 & 100,0 \\
\hline
\end{tabular}

casa. Quanto ao uso de álcool pelos pais, 70,8\% (503) relataram o uso por pelo menos um deles. Quando questionados com quem costumavam beber, $30,7 \%$ (218) dos jovens relataram que bebiam com a família, 34,5\% (245) com os amigos e $25,5 \%$ (181) somente experimentaram bebida alcoólica e hoje não bebem mais. Quanto aos problemas relacionados ao álcool, como brigas e absenteísmo escolar, 10,5\% (74) dos adolescentes relataram ter apresentado algum desses problemas nos últimos trinta dias (Tabela 3). A associação entre o uso de álcool pelos pais e o uso pelo menos uma vez na vida pelos escolares foi significativa $(\mathrm{p}<0,000)$. Encontrou-se $48 \%(\mathrm{RP}=$ 1,48; IC95\%: 1,31-1,66) a mais de experimentação da substância entre os jovens que relataram ter pais que fazem uso de bebida alcoólica do que entre aqueles cujos pais não bebem.

Entre os adolescentes estudados, 97,6\% relataram nunca ter feito uso de outras drogas. Entre os 28 que experimentaram, 67,9\% (19) tiveram a maconha como a droga da primeira experiência, seguidos de $10,7 \%$ (3) que utilizaram solventes, $7,1 \%$ (2) anabolizantes, 7,1\% (2) cocaína, 3,6\% (1) anfetaminas e $3,6 \%$ (1) crack. Quando questionados sobre o uso de outras drogas nos últimos trinta dias, 57,1\% (16) relataram não ter utilizado e $25 \%$ (7) mencionaram ter usado maconha. A experimentação dessas drogas foi 11,1 (IC95\%: $4,25-28,93)$ vezes maior entre os adolescentes que relataram o uso pelos amigos $(\mathrm{p}<0,000)$.

Em relação às variáveis sexo, cor da pele, classificação sócio-econômica e percepção da imagem corporal, não se encontrou associação com os desfechos investigados. Quando avaliado o estado nutricional, o uso de álcool apresentou uma prevalência $26 \%$ menor entre os adolescen- 
tes com sobrepeso/obesidade. Não foi possível realizar a análise bivariada em relação ao uso de outras drogas devido ao pequeno número de casos com esse desfecho.

Os jovens que referiram sentimentos de tristeza nos últimos 12 meses tiveram uma freqüência de uso de tabaco e de bebidas alcoólicas, 2,6 e 1,7 vezes maior, respectivamente, do que os que não se sentiram assim. Diferentemente, não se evidenciou essa associação em relação ao uso de outras drogas. Entre os adolescentes que se sentiram sozinhos nos últimos 12 meses, encontrou-se $87 \%$ a mais de tabagismo e $36 \%$ a mais de uso de bebida alcoólica do que seus pares. Essa variável apresentou comportamento semelhante quanto ao uso das outras drogas, tendo uma prevalência 6,4 vezes maior entre os que se sentiram sozinhos.

Em relação à qualidade do sono, o uso de tabaco e de álcool foi 2,2 e 1,3 vezes maior, respectivamente, entre os escolares com dificuldade de dormir. No entanto, não foi verificada associação estatisticamente significativa com o uso das outras drogas. Os que referiram ideação suicida no último ano tiveram uma prevalência aumentada de todos os desfechos, com três vezes mais tabagismo, cerca de duas vezes mais uso de álcool e seis vezes mais uso das outras drogas (Tabela 4).

\section{Discussão}

A amostra estudada pode ser considerada como representativa dos escolares da 7ạ série do ensino público de Gravataí, em função do processo de amostragem utilizado. Além disso, as perdas não comprometeram o tamanho da amostra, distribuindo-se igualmente entre os sexos e as regiões administrativas do município.

A baixa prevalência de consumo das drogas investigadas talvez possa ter sido provocada pela omissão dos escolares sobre suas experiências passadas. Por outro lado, não podemos esquecer que este estudo foi realizado no ambiente escolar, tendo esses adolescentes mais acesso a informações e, provavelmente, melhores condições de vida do que os evadidos do sistema. Justamente por esse motivo, escolheu-se esse grupo populacional, pois as ações de promoção, quando exercidas no ambiente escolar, podem provocar mudanças sociais, ambientais e no estilo de vida do adolescente, buscando uma educação para saúde com enfoque nas necessidades integrais do aluno 22 . Muitas das causas da morbimortalidade na idade adulta podem ser reduzidas por meio de políticas públicas de prevenção de doenças e promoção da saúde, desenvolvidas na puberdade.
Tabela 3

Distribuição dos escolares que fizeram uso de álcool na vida em relação ao local da primeira experiência e problemas relacionados ao uso da substância. Gravataí, Rio Grande do Sul,

Brasil, 2005.

\begin{tabular}{|c|c|c|}
\hline Variáveis & $\mathrm{n}$ & $\%$ \\
\hline \multicolumn{3}{|l|}{ Onde bebeu pela primeira vez } \\
\hline Em casa & 362 & 51,0 \\
\hline Escola & 2 & 0,3 \\
\hline Casa dos amigos & 100 & 14,1 \\
\hline Parque, rua e outros & 118 & 16,6 \\
\hline Bar, danceteria ou restaurante & 125 & 17,6 \\
\hline Ignorado & 3 & 0,4 \\
\hline \multicolumn{3}{|l|}{ Bebeu exageradamente } \\
\hline Nenhuma vez & 525 & 73,9 \\
\hline 1 ou 2 vezes & 167 & 23,5 \\
\hline $3-9$ vezes & 14 & 2,0 \\
\hline 10 ou mais vezes & 4 & 0,6 \\
\hline \multicolumn{3}{|l|}{ Reação da família } \\
\hline Não perceberia & 30 & 4,2 \\
\hline Perceberia, mas não se preocuparia & 19 & 2,7 \\
\hline Perceberia e ficaria chateada & 321 & 45,2 \\
\hline Não sabe & 340 & 47,9 \\
\hline \multicolumn{3}{|l|}{ Uso pelos pais } \\
\hline Não & 178 & 25,0 \\
\hline Sim, o pai & 257 & 36,2 \\
\hline Sim, a mãe & 41 & 5,8 \\
\hline Sim, os dois & 205 & 28,9 \\
\hline Não sabe & 29 & 4,1 \\
\hline \multicolumn{3}{|l|}{ Costuma beber } \\
\hline Não bebe & 181 & 25,5 \\
\hline Com os amigos & 245 & 34,5 \\
\hline Com a família & 218 & 30,7 \\
\hline Com pessoas que encontra ou sozinho & 29 & 4,1 \\
\hline Outros & 37 & 5,2 \\
\hline \multicolumn{3}{|l|}{ Problemas com os pais, brigas ou } \\
\hline \multicolumn{3}{|l|}{ ausência da escola nos últimos trinta dias } \\
\hline Nenhuma vez & 636 & 89,5 \\
\hline 1 ou 2 vezes & 53 & 7,5 \\
\hline 3 a 9 vezes & 19 & 2,7 \\
\hline 10 ou mais vezes & 2 & 0,3 \\
\hline Total & 710 & 100,0 \\
\hline
\end{tabular}

Os adolescentes estudados tiveram sua primeira experiência de uso de tabaco e álcool com idade média de 11,5 anos; a experimentação das outras drogas deu-se mais tarde, aos 13,0 anos. Semelhantemente, Ferigolo et al. 23, em estudo realizado em 1999, com jovens institucionalizados de Porto Alegre, mostram que a primeira experiência com o tabaco ou álcool ocorreu entre 10 e 12 anos de idade, enquanto com as drogas ilícitas, na faixa dos 13 e 15 anos. Outras 
Fatores associados ao uso de tabaco, álcool e outras drogas nos últimos trinta dias. Gravataí, Rio Grande do Sul, Brasil, 2005.

\begin{tabular}{|c|c|c|c|c|c|c|c|c|c|c|}
\hline \multirow[t]{2}{*}{ Varíaveis } & \multirow[t]{2}{*}{$\mathrm{n}$} & \multicolumn{3}{|c|}{ Tabaco } & \multicolumn{3}{|c|}{ Álcool } & \multicolumn{3}{|c|}{ Outras drogas } \\
\hline & & n (\%) & RP (IC95\%) & $p$ & n (\%) & RP (IC95\%) & $p$ & n (\%) & RP (IC95\%) & $\mathrm{p}$ \\
\hline \multicolumn{11}{|l|}{ Sexo } \\
\hline Masculino & 556 & $\begin{array}{c}25 \\
(4,5)\end{array}$ & $\begin{array}{c}1,02 \\
(0,60-1,74)\end{array}$ & 0,935 & $\begin{array}{c}186 \\
(33,4)\end{array}$ & $\begin{array}{c}1,03 \\
(0,87-1,20)\end{array}$ & 0,794 & $\begin{array}{c}3 \\
(0,5)\end{array}$ & $\begin{array}{c}0,83 \\
(0,19-3,69)\end{array}$ & 0,805 \\
\hline Feminino & 614 & $\begin{array}{l}27 \\
(4,4)\end{array}$ & 1,00 & - & $\begin{array}{l}200 \\
(32,6)\end{array}$ & 1,00 & - & $\begin{array}{c}4 \\
(0,6)\end{array}$ & 1,00 & - \\
\hline \multicolumn{11}{|l|}{ Cor da pele } \\
\hline Brancos & 615 & $\begin{array}{c}23 \\
(3,7)\end{array}$ & $\begin{array}{c}1,40 \\
(0,82-2,39)\end{array}$ & 0,221 & $\begin{array}{c}194 \\
(31,5)\end{array}$ & $\begin{array}{c}1,10 \\
(0,93-1,29)\end{array}$ & 0,268 & $\begin{array}{c}2 \\
(0,3)\end{array}$ & $\begin{array}{c}2,77 \\
(0,54-14,23)\end{array}$ & 0,222 \\
\hline Não brancos & 555 & $\begin{array}{l}29 \\
(5,2)\end{array}$ & 1,00 & - & $\begin{array}{c}192 \\
(34,6)\end{array}$ & 1,00 & - & $\begin{array}{c}5 \\
(0,9)\end{array}$ & 1,00 & - \\
\hline \multicolumn{11}{|l|}{ Classificação sócio-econômica } \\
\hline B & 256 & $\begin{array}{c}7 \\
(2,7)\end{array}$ & 1,00 & - & $\begin{array}{c}91 \\
(35,5)\end{array}$ & 1,00 & - & - & * & - \\
\hline C & 688 & $\begin{array}{c}31 \\
(4,5)\end{array}$ & $\begin{array}{c}1,64 \\
(0,73-3,70)\end{array}$ & 0,226 & $\begin{array}{c}208 \\
(30,2)\end{array}$ & $\begin{array}{c}0,85 \\
(0,70-1,04)\end{array}$ & 0,113 & $\begin{array}{c}5 \\
(0,7)\end{array}$ & * & - \\
\hline$E+D$ & 226 & $\begin{array}{c}14 \\
(6,2)\end{array}$ & $\begin{array}{c}2,26 \\
(0,93-5,51)\end{array}$ & 0,072 & $\begin{array}{c}87 \\
(38,5)\end{array}$ & $\begin{array}{c}1,08 \\
(0,86-1,37)\end{array}$ & 0,503 & $\begin{array}{c}2 \\
(0,9)\end{array}$ & * & - \\
\hline \multicolumn{11}{|l|}{ Percepção imagem corporal } \\
\hline Preocupado & 275 & $\begin{array}{c}15 \\
(5,4)\end{array}$ & $\begin{array}{c}1,31 \\
(0,73-2,35)\end{array}$ & 0,362 & $\begin{array}{c}95 \\
(34,5)\end{array}$ & $\begin{array}{c}1,08 \\
(0,89-1,30)\end{array}$ & 0,455 & $\begin{array}{c}1 \\
(0,4)\end{array}$ & $\begin{array}{c}0,54 \\
(0,07-4,46)\end{array}$ & 0,567 \\
\hline Não preocupado & 890 & $\begin{array}{c}37 \\
(4,1)\end{array}$ & 1,00 & - & $\begin{array}{c}287 \\
(32,2)\end{array}$ & 1,00 & - & $\begin{array}{c}6 \\
(0,7)\end{array}$ & 1,00 & - \\
\hline \multicolumn{11}{|l|}{ Estado nutricional } \\
\hline Eutrófico & 773 & $\begin{array}{c}37 \\
(4,8)\end{array}$ & 1,00 & - & $\begin{array}{c}277 \\
(35,8)\end{array}$ & 1,00 & - & $\begin{array}{c}7 \\
(0,9)\end{array}$ & * & - \\
\hline Risco nutricional e desnutrido & 157 & $\begin{array}{c}5 \\
(3,2)\end{array}$ & $\begin{array}{c}0,67 \\
(0,26-1,67)\end{array}$ & 0,385 & $\begin{array}{c}45 \\
(28,7)\end{array}$ & $\begin{array}{c}0,80 \\
(0,61-1,04)\end{array}$ & 0,098 & - & * & - \\
\hline Sobrepeso e obesos & 240 & $\begin{array}{c}10 \\
(4,2)\end{array}$ & $\begin{array}{c}0,87 \\
(0,44-1,72)\end{array}$ & 0,691 & $\begin{array}{c}64 \\
(26,7)\end{array}$ & $\begin{array}{c}0,74 \\
(0,59-0,94)\end{array}$ & 0,012 & - & * & * \\
\hline \multicolumn{11}{|l|}{$\begin{array}{l}\text { Sentimento de tristeza } \\
\text { nos últimos } 12 \text { meses }\end{array}$} \\
\hline $\operatorname{Sim}$ & 240 & $\begin{array}{l}21 \\
(8,8)\end{array}$ & $\begin{array}{c}2,62 \\
(1,54-4,48)\end{array}$ & 0,000 & $\begin{array}{c}116 \\
(48,3)\end{array}$ & $\begin{array}{c}1,66 \\
(1,41-1,96)\end{array}$ & 0,000 & $\begin{array}{c}3 \\
(1,2)\end{array}$ & $\begin{array}{c}2,90 \\
(0,65-12,90)\end{array}$ & 0,161 \\
\hline Não & 930 & $\begin{array}{c}31 \\
(3,3)\end{array}$ & 1,00 & - & $\begin{array}{c}270 \\
(29,0)\end{array}$ & 1,00 & - & $\begin{array}{c}4 \\
(0,4)\end{array}$ & 1,00 & - \\
\hline \multicolumn{11}{|l|}{$\begin{array}{l}\text { Sentimento de solidão } \\
\text { nos últimos } 12 \text { meses }\end{array}$} \\
\hline Sim & 329 & $\begin{array}{c}22 \\
(6,7)\end{array}$ & $\begin{array}{c}1,87 \\
(1,10-3,20)\end{array}$ & 0,022 & $\begin{array}{c}134 \\
(40,7)\end{array}$ & $\begin{array}{c}1,36 \\
(1,15-1,61)\end{array}$ & 0,000 & $\begin{array}{c}5 \\
(1,5)\end{array}$ & $\begin{array}{c}6,38 \\
(1,24-32,76)\end{array}$ & 0,026 \\
\hline Não & 840 & $\begin{array}{c}30 \\
(3,6)\end{array}$ & 1,00 & - & $\begin{array}{c}251 \\
(29,9)\end{array}$ & 1,00 & - & $\begin{array}{c}2 \\
(0,2)\end{array}$ & 1,00 & - \\
\hline \multicolumn{11}{|l|}{$\begin{array}{l}\text { Dificuldade de dormir } \\
\text { nos últimos } 12 \text { meses }\end{array}$} \\
\hline $\operatorname{Sim}$ & 223 & $\begin{array}{c}18 \\
(8,1)\end{array}$ & $\begin{array}{c}2,24 \\
(1,29-3,90)\end{array}$ & 0,004 & $\begin{array}{c}93 \\
(41,7)\end{array}$ & $\begin{array}{c}1,35 \\
(1,13-1,62)\end{array}$ & 0,001 & $\begin{array}{c}2 \\
(0,9)\end{array}$ & $\begin{array}{c}1,70 \\
(0,33-8,69)\end{array}$ & 0,526 \\
\hline Não & 946 & $\begin{array}{c}34 \\
(3,6)\end{array}$ & 1,00 & - & $\begin{array}{c}292 \\
(30,9)\end{array}$ & 1,00 & - & $\begin{array}{c}5 \\
(0,5)\end{array}$ & 1,00 & - \\
\hline \multicolumn{11}{|l|}{$\begin{array}{l}\text { Ideação suicida nos } \\
\text { últimos } 12 \text { meses }\end{array}$} \\
\hline Sim & 127 & $\begin{array}{c}15 \\
(11,8)\end{array}$ & $\begin{array}{c}3,33 \\
(1,88-5,89)\end{array}$ & 0,000 & $\begin{array}{c}70 \\
(55,1)\end{array}$ & $\begin{array}{c}1,82 \\
(1,52-2,18)\end{array}$ & 0,000 & $\begin{array}{c}3 \\
(2,4)\end{array}$ & $\begin{array}{c}6,16 \\
(1,40-27,23)\end{array}$ & 0,017 \\
\hline Não & 1.043 & $\begin{array}{c}37 \\
(3,5)\end{array}$ & 1,00 & - & $\begin{array}{c}316 \\
(30,3)\end{array}$ & 1,00 & - & $\begin{array}{c}4 \\
(0,4)\end{array}$ & 1,00 & - \\
\hline
\end{tabular}

* Não foi possível realizar a análise bivariada em virtude do pequeno número de usuários de drogas ilícitas. 
pesquisas, realizadas na cidade de Porto Alegre em anos anteriores, demonstraram a iniciação do uso dessas substâncias em uma idade mais avançada 9,24. Em estudo realizado por Souza \& Martins 25, em 1995, na cidade de Cuiabá, Mato Grosso, Brasil, as idades médias de uso inicial foram 12 anos para o álcool, 13 anos para o tabaco e 14 anos para drogas ilícitas. Outro trabalho realizado em Rosário, Argentina, em 1991, aponta o início do uso de tabaco com idade média de 16 anos 26 .

É possível que as diferenças ocorram por características tanto culturais, como temporais, uma vez que esses estudos foram realizados em outros locais, na década de 90, quando o consumo dessas substâncias era menos freqüente. Não existem dados anteriores disponíveis para o município estudado. No entanto, em face da semelhança entre a população estudada e os alunos do ensino público da Região Metropolitana de Porto Alegre, os resultados sugerem uma tendência ao uso de álcool, tabaco e outras drogas cada vez mais precocemente, reforçando a importância da orientação sobre os efeitos prejudiciais dessas substâncias no início da idade escolar, o que poderia prevenir a experimentação precoce e o uso abusivo na adolescência e nos adultos jovens.

O álcool apresentou-se como a substância de maior prevalência, seja como uso na vida, seja como nos últimos trinta dias. A crença de que a bebida alcoólica não é droga contribui para o estímulo e incentivo ao uso de álcool, não só durante a adolescência, mas em todas as faixas etárias. De acordo com o estudo divulgado pelo CEBRID 9, em estudantes dos ensinos fundamental e médio, a alta prevalência de uso na vida de bebida alcoólica é característica do país, variando de 46,1\% em Aracajú, Sergipe, Brasil, a $68,9 \%$ no Rio de Janeiro, Brasil. Isso aponta o consumo do álcool como um problema de saúde pública, principalmente na adolescência, pois o abuso dessa substância pode desencadear comportamentos violentos e acidentes de trânsito, que são as maiores causas de mortalidade nessa faixa etária.

A maioria dos adolescentes pesquisados relatou que a experimentação do álcool aconteceu junto à família e que costuma beber em casa e com os amigos. Também se observou maior consumo de álcool entre os escolares com histórico familiar de uso de álcool, semelhante ao citado por Souza et al. 27, em estudo realizado na cidade de Cuiabá. Essas situações familiares, aliadas ao fato de que $47,9 \%$ dos jovens que experimentaram álcool não sabiam qual seria a reação da família se chegassem embriagados em casa, são preocupantes, uma vez que evidenciam um ambiente familiar não protetor ao uso dessas subs- tâncias, podendo ser a família um facilitador à experiência e ao uso de álcool.

O Brasil é um dos principais produtores e exportadores de tabaco no mundo. Na Região Sul do país, onde estão concentradas as maiores indústrias fumageiras, as ações voltadas para o controle e a prevenção do tabagismo são dificultadas pelo poder econômico e político desse setor. A pesquisa realizada pelo Ministério da Saúde (VIGESCOLA), em 2004, abordando o tabagismo em escolares em 12 capitais brasileiras confirma essa realidade, indicando que $48,3 \%$ dos estudantes de Porto Alegre já haviam fumado cigarros, sendo a capital de maior prevalência de uso na vida de tabaco. Em Fortaleza, onde 20,5\% relataram fumar no momento da entrevista, verificouse a maior prevalência de fumantes atuais 11 .

O estudo de Gravataí mostrou que 16,9\% dos escolares experimentaram tabaco e $4,4 \%$ usaram nos últimos trinta dias, mantendo uma média de experimentação abaixo da encontrada pelo VIGESCOLA em Porto Alegre. No entanto, esse resultado foi semelhante ao divulgado pelo CEBRID 9 no VLevantamento Nacional sobre o Consumo de Drogas Psicotrópicas, em que a maior porcentagem de uso freqüente de tabaco foi $4,6 \%$, constatada na Região Sul.

A influência da família e dos amigos no consumo de tabaco pelos adolescentes também foi demonstrada. Quase a totalidade dos jovens que experimentaram tabaco relatou o uso dessa substância pelos amigos, semelhante aos estudos de Poletto et al. ${ }^{26}$ e Malcon et al. ${ }^{28}$, nos quais a maioria dos jovens citou o hábito de fumar dos amigos como motivo para a experimentação. Aqueles que relataram ter fumado nos últimos trinta dias afirmaram que o fazem em casa, podendo indicar que a família tem conhecimento dessa prática. Entre os que experimentaram tabaco, mais da metade relatou que pelo menos um dos pais é fumante, semelhante ao achado de outros autores 26,29. A pesquisa VIGESCOLA identificou que, em todas as capitais estudadas, a porcentagem dos escolares fumantes, que têm pelo menos um dos pais tabagista, excedeu a de escolares não fumantes na mesma condição ${ }^{11}$.

A transição saudável da infância para a adolescência ocorre por meio da negociação de novas regras e limites, baseada em uma relação de confiança e afeto entre pais e filhos ${ }^{4}$. A família é o primeiro grupo de referência na vida dos jovens 1 . Por isso, é fundamental que os pais possam se apresentar como modelos saudáveis, de modo que suas atitudes sejam coerentes com a orientação sobre os malefícios do cigarro e da bebida alcoólica 30 .

Como conseqüência da busca de independência e da formação da identidade, os amigos 
passam a exercer um papel cada vez mais importante na vida dos adolescentes. $\mathrm{O}$ excesso de conflitos intrafamiliares contribui para que o adolescente busque nas amizades relações de confiança ${ }^{4}$ que não conseguiu estabelecer em casa. Assim, para ser aceito pelo grupo, o jovem pode passar a fazer uso de álcool, tabaco e outras drogas, adquirindo comportamentos e atitudes semelhantes aos de seus pares. A escola é o local onde primeiramente ocorre a formação desses grupos, portanto é fundamental o papel que exerce no desenvolvimento de comportamentos e hábitos saudáveis nos adolescentes.

As outras drogas apresentaram baixa prevalência nesse estudo, talvez em virtude da faixa etária estudada, que foi de 14 anos em média, ou do medo dos estudantes em informar seu uso. Em estudo realizado por Guimarães et al. 31 , em adolescentes escolares de Assis, São Paulo, Brasil, as drogas ilícitas tiveram uma prevalência de uso de $19,7 \%$, permanecendo inferior ao álcool e tabaco. Entre os que experimentaram, a maconha foi a droga mais utilizada, seguida pelos solventes, semelhante a outros trabalhos 32,33 . Entretanto, diferentemente desses, outros autores 9,25,34 encontraram o solvente como a droga mais experimentada. É sabido que os usuários de outras drogas, na maioria das vezes, já utilizaram álcool e tabaco, logo é essencial que as ações para evitar o uso de drogas ilícitas e psicotrópicas sejam concomitantes às de combate ao fumo e ao uso de bebidas alcoólicas. Essa importante associação é apresentada por Arillo-Santillan et al. ${ }^{35}$, em estudo no México, onde o uso de tabaco esteve altamente relacionado ao uso de álcool e drogas ilícitas.

Em relação ao uso de álcool, tabaco e outras drogas nos últimos trinta dias, o sexo, a cor da pele e a classificação sócio-econômica não se mostraram associados. Em estudo realizado por Godói et al. 34, em estudantes da rede privada de Brasília, Brasil, o sexo masculino utilizou drogas ilícitas com maior freqüência. Silva et al. 33, investigando estudantes universitários do Município de São Paulo, identificaram que alunos com renda familiar alta apresentaram maior risco de consumo de drogas, porém também não evidenciaram associação com o sexo. Almeida-Filho et al. ${ }^{36}$ e Soldera et al. ${ }^{37}$ mostraram que o consumo de álcool está relacionado às classes sociais mais elevadas. Diferentemente, Muza et al. 38, em pesquisa na cidade de Ribeirão Preto, São Paulo, não encontraram diferenças na distribuição do consumo de álcool e tabaco entre as classes sociais.

O comportamento feminino em relação ao uso de substâncias lícitas e ilícitas tornou-se bastante semelhante ao dos homens nos últimos anos, principalmente na adolescência, uma vez que fazem parte de um grupo de iguais. Assim, existe uma tendência em adotarem os mesmos comportamentos, buscando a aceitação pelo próprio grupo. Quanto à situação sócio-econômica, a amostra estudada era bastante homogênea, uma vez que todos eram de escolas públicas municipais, não havendo nenhum aluno pertencente à classe A. Talvez isso tenha contribuído para a não associação do consumo das substâncias estudadas e a inserção sócio-econômica.

Entre os adolescentes, é comum a distorção da imagem corporal, evoluindo, algumas vezes, para o aparecimento de transtornos alimentares. Strauss \& Mir 39, em 2001, nos Estados Unidos, identificaram o uso de tabaco por adolescentes eutróficos na tentativa de perderem peso. Outro estudo 40, em 2006, também nos Estados Unidos, verificou que os transtornos alimentares estão associados ao uso de álcool e drogas ilícitas. Nessa fase da vida, a aceitação do grupo passa a ter papel extremamente relevante, pois um adolescente integrado ao grupo tende a se aceitar melhor. Com a ingestão de bebida alcoólica, o adolescente adquire uma falsa segurança, passando a uma aceitação temporária do seu corpo e sentindo-se mais corajoso para o enfrentamento de dificuldades. Contudo, no presente estudo, não se verificou associação estatisticamente significativa entre os desfechos e a percepção da imagem corporal. Além disso, com relação ao estado nutricional, os escolares com sobrepeso/ obesidade apresentaram menor prevalência de uso de álcool. É possível que os jovens com mais peso controlem o consumo de álcool como medida dietética, evitando um aumento do aporte calórico. Por outro lado, também é possível que a forma encontrada para o alívio de sua insegurança seja a ingesta aumentada de alimentos e não o consumo das substâncias investigadas. Entretanto, essa situação necessita ser mais bem estudada em pesquisas delineadas especialmente para este fim.

Os sentimentos de tristeza, solidão, e a dificuldade para dormir foram utilizados como possíveis sintomas de depressão, tendo-se verificado associação com o uso de álcool e tabaco; especificamente, a dificuldade de dormir mostrou associação com o uso de drogas ilícitas. Outros autores 41,42 também encontraram o uso de álcool associado à depressão. Jovens com sintomas depressivos apresentaram o dobro de chance de uso de álcool quando comparados àqueles sem nenhum sintoma de depressão no estudo de Wu et al. 43. Malbergier \& Oliveira Jr. ${ }^{44}$, em revisão de literatura, encontraram, em diversos artigos, a associação entre tabagismo e depressão. Da mesma forma, a Associação Brasileira de Psiquiatria 45, também em artigo de revisão, 
aponta que há poucas evidências de associação entre o uso infreqüente da maconha e depressão, porém afirma que o uso pesado dessa substância está associado. Isso não foi possível constatar em nosso estudo, pois somente sete adolescentes relataram o uso freqüente de outras drogas nos últimos trinta dias.

A ideação suicida também pode ser um indicativo de depressão, embora, na adolescência, muitos jovens pensem assim, sem desenvolver a doença. Os conflitos familiares, os problemas no relacionamento amoroso e a morte de pessoas queridas fazem com que o adolescente tenha esses pensamentos. Esse quadro reforça a importância da estrutura familiar, servindo de apoio no enfrentamento dessas situações. Os jovens que referiram pensamentos suicidas no último ano tiveram prevalência aumentada dos três desfechos. Resultado semelhante foi encontrado por Marcondes Filho et al. ${ }^{46}$, em estudo que registrou o uso de maconha, cocaína, solventes, álcool e tabaco entre jovens com tentativas prévias de suicídio, atendidos em um hospital universitário de Londrina, Paraná, Brasil. Esses resultados reforçam a existência de comorbidades associadas a sintomas depressivos, para os quais o uso de álcool, tabaco e drogas ilícitas acontece como forma de alívio.

\section{Considerações finais}

O presente estudo possibilitou a identificação da alta prevalência da experimentação de drogas entre escolares, principalmente do álcool. Também se verificou uma redução dos jovens que fizeram uso das substâncias nos últimos trinta dias em relação aos que utilizaram pelo menos uma vez na vida, indicando, talvez, a inexistência do há- bito consolidado. Isso aponta para a importância da detecção precoce de jovens mais vulneráveis, possibilitando o desenvolvimento de ações para que sejam prevenidos o abuso e a dependência dessas substâncias.

As ações de promoção da saúde devem ser direcionadas também à família, já que esta exerce importante influência no comportamento do indivíduo na fase da adolescência, servindo como modelo. Para tanto, é necessária a ação interdisciplinar e intersetorial, proporcionando trabalho integrado entre os diversos setores envolvidos.

As Escolas Promotoras da Saúde fazem parte das estratégias de saúde coletiva utilizadas mundialmente, deslocando o foco do indivíduo para os atores coletivos 47 . Nesse sentido, assume-se uma visão integral do ser humano, considerando o adolescente em seu ambiente familiar, comunitário e social, promovendo o desenvolvimento de conhecimentos e habilidades para o seu autocuidado, a autonomia, a criatividade e a participação do jovem na construção de sua cidadania 48 . Além disso, o ambiente escolar também deve ser um local de hábitos saudáveis, onde são incentivados novos comportamentos, como a alimentação adequada, a prática de atividade física e o não-uso de álcool, tabaco e drogas ilícitas 49. Conseqüentemente, na escola, os jovens encontrarão outros jovens com comportamentos semelhantes, formando grupos de amigos com atitudes positivas para a manutenção da saúde.

Os profissionais de saúde devem estar inseridos nesse contexto, trabalhando conjuntamente com a escola na elaboração de atividades de educação em saúde, direcionadas ao adolescente e sua família, e no planejamento de políticas voltadas à construção de ambientes protetores e saudáveis para melhorar a qualidade de vida dos alunos e da comunidade. 


\section{Resumo}

Este trabalho teve como objetivo estudar o comportamento dos estudantes do ensino público municipal de Gravataí, Rio Grande do Sul, Brasil, em relação ao consumo de álcool, tabaco e outras drogas, investigando fatores associados. Foi utilizado delineamento transversal com amostra aleatória representativa de 1.170 escolares da $7 a$ série e estratificada por região do município. As associações entre desfechos e fatores em estudo foram testadas com regressão de Cox bivariada, modificada para estudos transversais. Foram encontrados, respectivamente, $60,7 \%, 16,9 \%$ e 2,4\% de uso na vida de bebida alcoólica, tabaco e outras drogas. Nos últimos trinta dias, o álcool também apresentou a maior prevalência (33\%), seguido pelo tabaco $(4,4 \%) e$ outras drogas $(0,6 \%)$. O estudo revelou a importância da família e dos amigos na experimentação das substâncias pelos jovens. A prevalência do uso de tabaco e álcool nos últimos trinta dias esteve associada à presença de sentimentos de tristeza, solidão, dificuldade para dormir e ideação suicida. O consumo de outras drogas esteve associado ao sentimento de solidão e ideação suicida.

Comportamento do Adolescente; Bebidas Alcoólicas; Tabaco; Drogas Ilícitas

\section{Referências}

1. Saito MI. Adolescência, cultura, vulnerabilidade e risco. Pediatria (São Paulo) 2000; 22:217-9.

2. Jerusalinsky A. Adolescência e contemporaneidade. In: Mello A, Castro ALS, Geiger M, organizadores. Conversando sobre adolescência e contemporaneidade. Porto Alegre: Editora Libretos; 2004. p. 54-65.

3. Coordenação Geral de Doenças e Agravos Não Transmissíveis, Secretaria de Vigilância em Saúde, Ministério da Saúde. Pesquisa Nacional de Saúde do Escolar - PeNSE. Brasília: Ministério da Saúde; 2006.

4. Schenker M, Minayo MCS. A implicação da família no uso abusivo de drogas: uma revisão crítica. Ciênc Saúde Coletiva 2003; 8:299-306.

5. Tavares BF, Béria JU, Lima MS. Fatores associados ao uso de drogas entre escolares. Rev Saúde Pública 2004; 38:787-96.

6. Ayres JRCM, França Júnior I, Calazans GJ. O conceito de vulnerabilidade e as práticas de saúde: novas perspectivas e desafios. In: Czeresnia D, Freitas CM, organizadores. Promoção da saúde: conceitos, reflexões, tendências. Rio de Janeiro: Editora Fiocruz; 2003. p. 117-39.

\section{Colaboradores}

P. C. Vieira participou de todas as etapas, desde a coleta e análise dos dados até a redação final do artigo. D. R. G. C. Aerts colaborou em todas as etapas do estudo, desde o seu planejamento até a análise dos dados e redação final do artigo. S. L. Freddo e A. Bittencourt participaram da coleta dos dados e redação do artigo. L. Monteiro contribuiu no planejamento do estudo, coleta dos dados e redação final do artigo.
7. Tedesco S. Adolescência e drogas: algumas indicações éticas e políticas. In: Mello A, Castro ALS, Geiger M, organizadores. Conversando sobre adolescência e contemporaneidade. Porto Alegre: Editora Libretos; 2004. p. 106-11.

8. World Health Organization. Overview of child and adolescent health: adolescent health and development. http://www.who.int/child-adolescenthealth/OVERVIEW/AHD/adh_over.htm (acessado em Ago/2006).

9. Centro Brasileiro de Informações sobre Drogas Psicotrópicas. V levantamento nacional sobre o consumo de drogas entre estudantes do ensino fundamental e médio da rede pública de ensino nas 27 capitais brasileiras - 2004. http://www. unifesp.br/dpsicobio/cebrid/levantamento_ brasil2/index.htm (acessado em Jun/2005).

10. Pechansky F, Barros F. Problems related to alcohol consuption by adolescents living in the city of Porto Alegre, Brazil. J Drug Issues 1995; 25:735-40. 
11. Instituto Nacional de Câncer. Vigescola: vigilância de tabagismo em escolares. Dados e fatos de 12 capitais brasileiras. v. 1. http://www.inca.gov.br/ vigescola/docs/vigescola_completo.pdf (acessado em Mai/2005).

12. Sanceverino SL, Abreu JLC. Aspectos epidemiológicos do uso de drogas entre estudantes do ensino médio no município de Palhoça, 2003. Ciênc Saúde Coletiva 2004; 9:1047-56.

13. World Health Organization. Chronic diseases and health promotion: global school-based student health survey (GSHS). http://www.who.int/chp/ gshs/en/index.html (acessado em Ago/2005).

14. World Health Organization. Chronic diseases and health promotion: global school-based student health survey (GSHS) purpose and methodology. http:/ / www.who.int/chp/gshs/methodology/en/ index.html (acessado em Ago/2005).

15. World Health Organization. Physical status: the use and interpretation of antropometric. Geneva: World Health Organization; 1995.

16. Must A, Dallal GE, Dietz WH. Reference data for obesity: 85th and 95th percentiles of body mass index (wt/ht2) and triceps skinfold thickness. Am J Clin Nutr 1991; 53:839-46.

17. Cole TJ, Bellizzi MC, Flegal KM, Dietz WH. Establishing a standard definition for child overweight and obesity worldwide: international survey. BMJ 2000; 320:1240-3.

18. Vitollo MR. Nutrição: da gestação a adolescência. Rio de Janeiro: Reichmann \& Affonso Editores; 2003.

19. Cooper P, Taylor MJ, Cooper Z, Fairburn CG. The development and validation of the Body Shape Questionnaire. Int J Eat Disord 1987; 6:485-94.

20. Cordás TA, Castilho SM. Imagem corporal nos transtornos alimentares. Instrumentos de avaliação: Body Shape Questionnaire. Psiquiatr Biol 1994; 2:17-21.

21. Barros A, Hirakata V. Alternatives for logistic regression in cross-sectional studies: an empirical comparison of models that directly estimate the prevalence ratio. BMC Med Res Methodol 2003; 3:1-13.

22. Ippolito-Shepherd J. A promoção da saúde no âmbito escolar: a iniciativa regional Escolas Promotoras da Saúde. Cadernos de Escolas Promotoras de Saúde - I. http://www.sbp.com.br/img/cadernos bpfinal.pdf (acessado em Nov/2006).

23. Ferigolo M, Barbosa FS, Arbo E, Malysz AS, Stein AT, Barros HMT. Prevalência do consumo de drogas na FEBEM, Porto Alegre. Rev Bras Psiquiatr 2004; 26:10-6.

24. Pechansky F, Inciardi JA, Surratt H, Lima AFBS, Kessler FP, Soibelman M, et al. Estudo sobre as características de usuários de drogas injetáveis que buscam atendimento em Porto Alegre, RS. Rev Bras Psiquiatr 2000; 22:164-71.

25. Souza DPO, Martins DTO. O perfil epidemiológico do uso de drogas entre estudantes de 1o e 2 o graus da rede estadual de ensino de Cuiabá, Brasil, 1995. Cad Saúde Pública 1998; 14:391-400.

26. Poletto L, Pezzotto SM, Morini J, Andrade J. Prevalencia del habito de fumar en jóvenes y sus padres. Asociaciones relevantes con educacion y ocupación. Rev Saúde Pública 1991; 25:388-93.
27. Souza DPO, Areco KN, Silveira Filho DX. Álcool e alcoolismo entre adolescentes da rede estadual de ensino de Cuiabá, Mato Grosso. Rev Saúde Pública 2005; 39:585-92.

28. Malcon MC, Menezes AMB, Maia MFS, Chatkin M, Victora CG. Prevalência e fatores de risco para tabagismo em adolescentes na América do Sul: uma revisão sistemática da literatura. Rev Panam Salud Pública 2003; 13:222-8.

29. Bolzán A, Peleteiro R. Tabaquismo durante la adolescencia temprana. Estudio en escolares argentinos. J Pediatr (Rio J) 2003; 79:461-6.

30. Ruiz MR, Andrade D. La familia y los factores de riesgo relacionados con el consumo de alcohol y tabaco en los niños y adolescentes (Guayaquil-Ecuador). Rev Latinoam Enferm 2005; 13(n. esp):813-8.

31. Guimarães JL, Godinho PH, Cruz R, Kappann JI, Tosta Junior LA. Consumo de drogas psicoativas por adolescentes escolares de Assis, SP. Rev Saúde Pública 2004; 38:130-2.

32. Baus J, Kupek E, Pires M. Prevalência e fatores de risco relacionados ao uso de drogas entre escolares. Rev Saúde Pública 2002; 36:40-6.

33. Silva LVER, Malbergier A, Stempliuk VA, Andrade AG. Fatores associados ao consumo de álcool e drogas entre estudantes universitários. Rev Saúde Pública 2006; 40: 280-8.

34. Godói AMM, Muza GM, Costa MP, Gama MLT. Consumo de substâncias psicoativas entre estudantes de rede privada. Rev Saúde Pública 1991; 25:150-6.

35. Arillo-Santillan E, Lazcano-Ponce E, Hernandez-Avila M, Fernández E, Allen B, Valdes R, et al. Associations between individual and contextual factors and smoking in 13,293 Mexican students. Am J Prev Med 2005; 28:41-51.

36. Almeida-Filho N, Lessa I, Magalhães L, Araújo MJ, Aquino E, Kawachi I, et al. Alcohol drinking patterns by gender, ethnicity, and social class in Bahia, Brazil. Rev Saúde Pública 2004; 38:45-54.

37. Soldera M, Dalgalarrondo P, Corrêa Filho HR, Silva CAM. Uso pesado de álcool por estudantes dos ensinos fundamental e médio de escolas centrais e periféricas de Campinas (SP): prevalência e fatores associados. Rev Bras Psiquiatr 2004; 26:174-9.

38. Muza GM, Bettiol H, Muccillo G, Barbieri MA. Consumo de substâncias psicoativas por adolescentes escolares de Ribeirão Preto, SP (Brasil). II - Distribuição do consumo por classes sociais. Rev Saúde Pública 1997; 31:163-70.

39. Strauss RS, Mir HM. Smoking and weight loss attempts in overweight and normal-weight adolescents. Int J Obes Relat Metab Disord 2001; 25:1381-5.

40. Conason AH, Sher L. Alcohol use in adolescents with eating disorders. Int J Adolesc Med Health 2006; 17:31-6.

41. Soares KVS, Busnello EA, Coutinho ES, AlmeidaFilho N, Andreoli SB, Mari JJ. Comorbidade nos transtornos depressivos na população de três centros urbanos brasileiros. Neurobiologia 1995; 58:77-86. 
42. Ratto LRC. Prevalência da comorbidade entre transtornos mentais graves e transtornos devido ao uso de substâncias psicoativas em São Paulo, Brasil [Dissertação de Mestrado]. São Paulo: Faculdade de Medicina, Universidade de São Paulo; 2000.

43. Wu P, Bird HR, Liu X, Fan B, Fuller C, Shen S, et al. Childhood depressive symptoms and early onset of alcohol use. Pediatrics 2006; 118:1907-15.

44. Malbergier A, Oliveira Jr. HP. Dependência de tabaco e comorbidade psiquiátrica. Rev Psiquiatr Clín (São Paulo) 2005; 32:276-82.

45. Associação Brasileira de Psiquiatria. Uso de maconha e doenças psiquiátricas. http://www.abpbrasil. org.br/departamentos/coordenadores/coorde nador $/$ noticias $/$ ?not $=88 \&$ dep=62 (acessado em Nov/2006)
46. Marcondes Filho W, Mezzaroba L, Turini CA, Koike A, Motomatsu Junior A, Shibayama EEM, et al. Tentativas de suicídio por substâncias químicas na adolescência e juventude. Adolesc Latinoam 2002; 3:0-0.

47. Organización Panamericana de la Salud. Escuelas promotoras de la salud: entornos saludables y mejor salud para las generaciones futuras. Washington DC: Organización Panamericana de la Salud; 1998.

48. Harada J. Introdução. Cadernos de Escolas Promotoras de Saúde - I. http://www.sbp.com.br/img/ cadernosbpfinal.pdf (acessado em Nov/2006).

49. Silva CS. Escola Promotora de Saúde: uma visão crítica da saúde escolar. Cadernos de Escolas Promotoras de Saúde - I. http://www.sbp. com.br/img/cadernosbpfinal.pdf (acessado em Nov/2006).

Recebido em 29/Mai/2007

Versão final reapresentada em 02/Abr/2008

Aprovado em 16/Abr/2008 